SCIENTIFIC REPORT

\title{
The effect of image alignment on capillary blood flow measurement of the neuroretinal rim using the Heidelberg retina flowmeter
}

\author{
M Sehi, J G Flanagan
}

Br J Ophthalmol 2004;88:204-206. doi: 10.1136/bjo.2003.020321

\begin{abstract}
Aim: To examine the influence of image alignment on the repeatability of blood flow measurements of the optic nerve. Methods: 10 normal subjects were examined. Heidelberg retina tomograph imaging was performed to establish best location and focus for the temporal neuroretinal rim. Two high quality Heidelberg retina flowmeter (HRF) images were acquired for three methods of alignment: central, nasal, and temporal. A $10 \times 10$ pixel measurement window was selected and exactly reproduced on all images. The interquartile pixel values were used to calculate capillary flow. ANOVA, intraclass correlation coefficients (ICC) and the coefficient of repeatability (CoR) were used for analysis.

Results: There was no difference between methods ( $p=0.47$ ) or between visits $(p=0.51)$. The ICCs were 0.83 for the central, 0.34 for the nasal, and 0.42 for the temporal alignment. The CoR was 31.5 for central (mean effect 235.1), 234.6 for nasal, and 256.7 for temporal alignment. Conclusion: Central alignment was the most repeatable method for the measurement of neuroretinal rim capillary blood flow using the HRF.
\end{abstract}

$\mathrm{T}$ he Heidelberg retina flowmeter (HRF, Heidelberg Engineering GmbH, Germany) is a scanning laser Doppler device used to estimate the ocular capillary blood flow. ${ }^{1-6}$ Acquisition parameters influence the variability of flow values. ${ }^{6-11}$ A recent study suggested placing the temporal rim margin tangent to the lateral image border for more reproducible measurement of the neuroretinal rim. ${ }^{11}$ The purpose of this study was to examine the influence of different image alignments on the repeatability of blood flow measurements of the neuroretinal rim using the HRF.

\section{METHODS}

Institutional ethics approval was granted. Imaging was performed on one randomly selected eye of 10 normal subjects (age range 23-44 years, mean 29.9 (SD 7.6), four females, corrected $V A \geqslant 6 / 9$ ).

Heidelberg retina tomograph (HRT) imaging was performed and the profile map used to determine the dioptric difference between the peripapillary retinal surface and the temporal rim. The best dioptric focus for the temporal rim was used for HRF imaging ${ }^{12}$ using three different methods of alignment:

- central: the optic nerve head was positioned at the centre of the image;

- nasal: the nasal rim margin was placed tangent to the nasal side of the image;
- temporal: the temporal rim margin was placed tangent to the temporal side of the image.

The sensitivity was optimised for the temporal neuroretinal rim while avoiding oversaturation. One investigator performed all the measurements.

Images were included if they had an average DC value of $\geqslant 175$ at the retina, and no saccades greater than $30 \mathrm{~ms}$.

A $10 \times 10$ pixel measurement window was placed on the temporal neuroretinal rim of a central alignment image, avoiding major vessels. ${ }^{2}$ The image was then printed onto a transparency and placed on the computer monitor. The window was exactly reproduced for all images (fig 1). One hundred individual pixel values were extracted for each window. The upper and lower 25th percentile of flow values were excluded to reduce the outliers that may have been caused by, for example, heart beat or level of image saturation. The mean flow values of the remaining interquartile range were analysed using ANOVA to compare between alignments and across the two visits $(\mathrm{p}<0.05)$. The intraclass correlation coefficients (ICCs) and the coefficients of repeatability (CoR) were calculated to assess concordance and repeatability. ${ }^{13}$

\section{RESULTS}

There was no significant difference between alignments or visits for the mean ocular capillary blood flow (table 1). The mean and median of the original 100 pixel values within the $10 \times 10$ pixel window were compared to the mean of the interquartile range. There was a significant difference in the means of all alignments and visits $(\mathrm{p}<0.001)$. There was no significant difference for the median for central alignment (visit $1 ; \mathrm{p}=0.13$; visit $2 ; \mathrm{p}=0.53$ ).

The mean of the differences (MoD), CoRs, and ICCs for the mean of the interquartile range of flow values between visits are listed in table 2. Central alignment had the smallest MoD (4.3) and CoR (31.5) and temporal alignment had the largest MoD (81.6) and CoR (256.7).

These results were supported by the ICCs which were "almost perfect" at 0.83 for central, "fair" at 0.34 for nasal, and "moderate" at 0.42 for temporal alignment. ${ }^{14}$

\section{DISCUSSION}

We were interested in finding the most repeatable method for the measurement of ocular capillary blood flow of the neuroretinal rim. In particular we wanted to investigate the assertion that decentration of the optic nerve, such that the temporal aspect of the optic nerve head was positioned tangential to the edge of the image, improved the repeatability of the HRF measurements. ${ }^{11}$ We found that the central alignment technique, with careful consideration of focal plane, image quality, and detector sensitivity, gave the most repeatable results when compared to nasal and temporal 

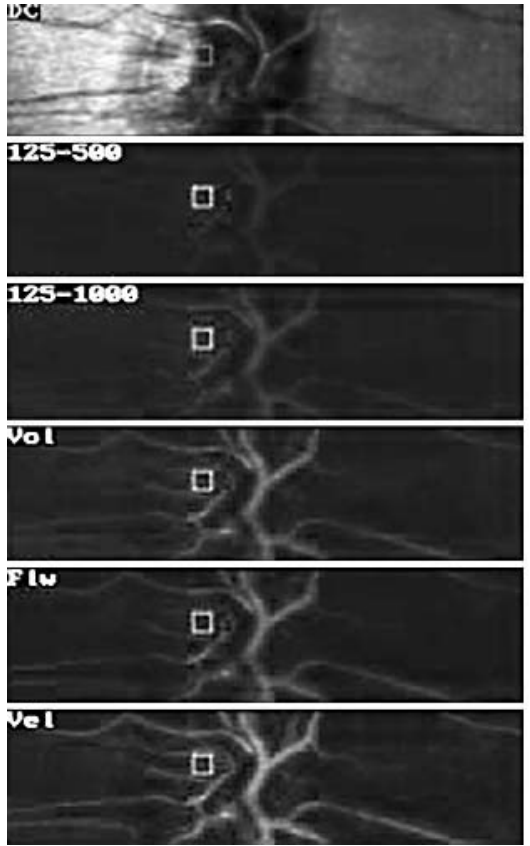
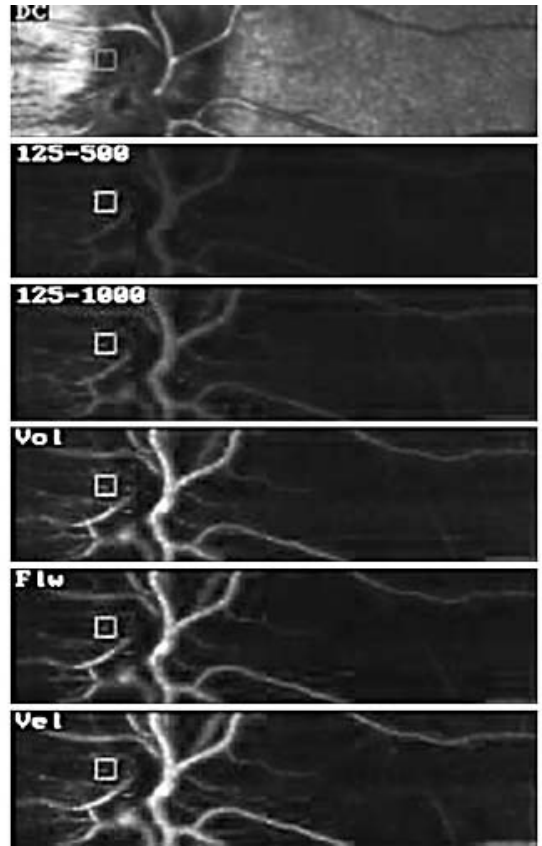
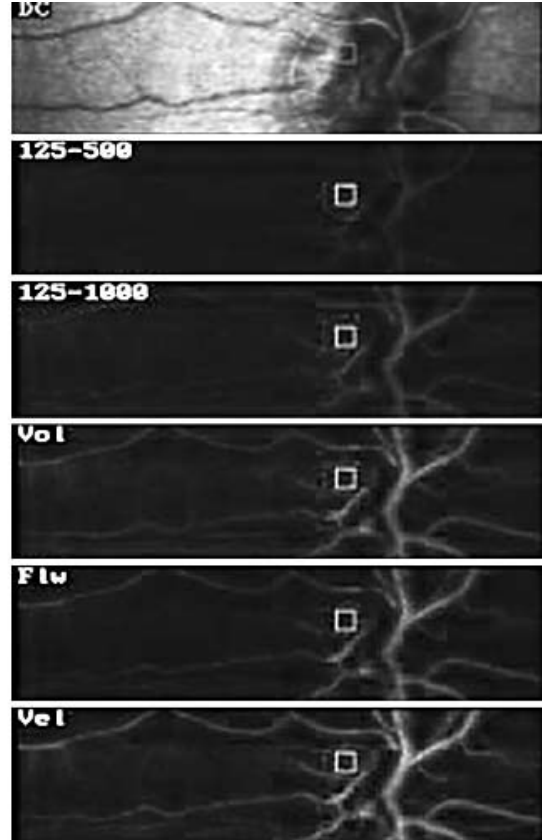

Figure 1 Heidelberg retina tlowmeter measurements were performed using three different methods of alignment: central, temporal, and nasal. The $10 \times 10$ pixel window was exactly aligned for three different methods.

Table 1 The mean and standard deviation (SD) of the middle $50 \%$ of ocular capillary blood flow values at two visits

\begin{tabular}{llll}
\hline Visit & Alignment & Range of means (group mean) & Range of SDs (group SD) \\
\hline 1 & Central & $91.6-458.9(230.8)$ & $82.7-272.1(125.9)$ \\
& Nasal & $62.0-422.3(251.5)$ & $89.8-255.4(91.8)$ \\
& Temporal & $23.9-248.6(154.9)$ & $48.4-212.5(84.7)$ \\
2 & Central & $97.4-469.8(235.1)$ & $84.9-276.1(132.9)$ \\
& Nasal & $73.8-419.9(228.3)$ & $78.6-165.8(118.7)$ \\
& Temporal & $41.1-559.3(236.5)$ & $71.0-184.0(156.8)$ \\
\hline
\end{tabular}

alignment techniques. The difference in results was predictable given the methodologies used. Jonescu-Cuypers et $a l^{11}$ measured the entire rim using a focus setting that was optimal for the retina, rather than the rim tissue, but their temporal alignment technique measured the rim with increased photodetector sensitivity compared to their central alignment technique.

In the majority of previous ocular flow studies using the HRF, the image was focused on the peripapillary retina when analysing the neuroretinal rim. Implicit within such a methodology is that the area of interest-that is, the neuroretinal rim, will be outside the optimal focal plane for the flowmetry measurements, a critical aspect of the technique. ${ }^{21115}$ A recent study by Hafez et $a l^{16}$ confirmed that the location of the focal plane has an impact on the measured flow values and that the HRT images were useful in finding the optimal focal plane for the flowmetry of the neuroretinal rim. Also image saturation is a limiting factor for the operator that helps to acquire more repeatable images. The large difference between visits for the temporal alignment technique was consistent with the inherently large variability most likely caused by difficulties in judging image saturation. It has previously been suggested that two factors that decrease the variability of the neuroretinal rim flow values are photodetector sensitivity adjustment and the inclusion of single pixel values in the flow analysis. ${ }^{11}{ }^{17}$ We suggest image focus and image saturation as additional factors.

Different attempts have been made to increase the sampling size of the flow values to reduce the variability of the measurements. ${ }^{6}{ }^{18}$ The pointwise analysis of the entire

Table 2 Mean of differences (MOD), coefficient of repeatability (CoR), and intraclass correlation coefficient (ICC) of ocular capillary blood flow values for each method of alignment

\begin{tabular}{lccl}
\hline Alignment & MoD (SD) & CoR (mean effect) & ICC of flow values \\
\hline Central & $4.3(16.1)$ & $31.5(235.1)$ & 0.83 \\
Nasal & $-23.2(119.7)$ & $234.6(228.3)$ & 0.34 \\
Temporal & $81.6(131.0)$ & $256.7(236.5)$ & 0.42 \\
\hline
\end{tabular}


image has been considered to provide additional power for the analysis of flow values and increase the long term reproducibility. ${ }^{15}{ }^{16}$ However, it may not be appropriate for smaller areas of interest. Additionally, when the retinal plane is in focus and the rim area remains unfocused and dark, the proportion of outlier flow values from the rim that are excluded from the analysis would be high. Hosking et al introduced a strategy in which a $10 \times 10$ pixel frame was repositioned within a $15 \times 15$ window and 36 mean flow values were extracted and ranked to find the highest and lowest local values of blood flow. The intrinsic effect of saturation and pulse on the maximum and minimum flow values is not excluded in this method.

We used the interquartile range for flow values within the measurement window to reduce the effect of variation as a result of underexposure and oversaturation of the image, and the cardiac cycle. ${ }^{19}{ }^{20}$ The HRF software generates the mean of data points within a measurement window. However, we found that in many cases the lower quartile of values consisted mainly of zeroes. Therefore the data were not Gaussian in distribution. The mean of such a distribution would result in a biased description of central tendency. In these cases the median is a better central descriptor. However, the median does not take into account the spread of data around the central measure. The interquartile range is a better descriptor of the spread and the central tendency of the data. The skewness of data was reduced by removing the zeroes, very low and very high values, as a result of undersaturation and oversaturation, and/or heart beat. Therefore, the distribution of the flow values was more Gaussian and the mean of the interquartile range and the median of the whole 100 data points were not significantly different. We therefore recommend that if a single average value for a measurement window is required then the median average of all pixels would be appropriate, but that if an estimate of the variance within the data is required the interquartile range and its mean would be more appropriate.

The "automatic full field perfusion image analyser" (AFFPIA) is a novel tool designed for the analysis of the retinal microvasculature flow. ${ }^{21} 22$ It provides an overall analysis of the rim and analysis of the average flow within a measurement window after correcting for invalid DC values, large vessels, and saccades. However, we were unable to use AFFPIA for this study as it does not provide the flow values for each pixel within a measurement window, and does not permit the accurate placement of a measurement window on subsequent images of the same optic nerve head.

Our protocol for assessment of the capillary blood flow in a small area of the neuroretinal rim incorporates three essential aspects including previous imaging with the HRT to establish precise focus of the rim and peripapillary retina; central alignment of the optic nerve head during image acquisition; and point by point analysis of the interquartile range of flow values within the measurement window.

\section{ACKNOWLEDGEMENT}

Financial support was provided by Glaucoma Research Society of Canada.

\section{Sehi, J G Flanagan, School of Optometry University of Waterloo, Waterloo, ON, Canada N2L 3G1}

Correspondence to: Dr Mitra Sehi, School of Optometry University of Waterloo, Waterloo, ON, Canada N2L 3G1;

msehizad@sciborg.uwaterloo.ca

Accepted for publication 2 July 2003

\section{REFERENCES}

1 Schuman JS. Imaging in glaucoma. Thorofare, NJ: Slack, 1996.

2 Zinser G: Scanning laser Doppler flowmetry. Principle and technique. In: Pillunat LE, Harris A, Anderson DR, Greve EL, eds. Current concepts on ocular blood flow in glaucoma. The Hague: Kugler Publications, 1999:197-204.

3 Flanagan JG. Imaging of the optic nerve and nerve fibre layer in glaucoma. In: Fingeret $M$, Lewis TL, eds. Primary care of the glaucomas. New York: McGraw-Hill, 2001:187-200.

4 Michelson G, Schmauss B. Two dimensional mapping of the perfusion of the retina and optic nerve head. Br J Ophthalmol 1995;79:1126-32.

5 Michelson G, Schmauss B, Langhans MJ, et al. Principle, validity, and reliability of scanning laser Doppler flowmetry. J Glaucoma 1996;5:99-105.

6 Kagemann L, Harris A, Chung HS, et al. Photodetector sensitivity level and Heidelberg retina flowmeter measurements in humans. Invest Ophthalmol Vis Sci 2001;42:354-7.

7 Bohdanecka Z, Orgul S, Prunte C, et al. Influence of acquisition parameters on hemodynamic measurements with the Heidelberg retina flowmeter at the optic disc. J Glaucoma 1998;7:151-7.

8 Hosking SL, Embleton SJ, Cunliffe IA. Application of a local search strategy improves the detection of blood flow deficits in the neuro-retinal rim of glaucoma patients using scanning laser Doppler flowmetry. Br J Ophthalmol 2001;85:1298-302.

9 Hosking SL, Embleton S, Kagemann L, et al. Detector sensitivity influences blood flow sampling in scanning laser Doppler flowmetry. Graefes Arch Clin Exp Ophthalmol 2001;239:407-10.

10 Rawii MH, Flanagan JG. Intraocular and interocular symmetry in normal retinal capillary perfusion. J Glaucoma $2001 ; 10: 4-12$.

11 Jonescu-Cuypers CP, Chung HS, Kagemann L, et al. New neuroretinal rim blood flow evaluation method combining Heidelberg Retina flowmetry and tomography. Br J Ophthalmol 2001;85:304-9.

12 Prokopich CL, Lundmark PO, Flanagan JG. Determination of optimum focus for the Heidelberg Retina Flowmeter using the Heidelberg retina tomograph. Invest Ophthalmol Vis Sci 2000;41:2954.

13 Bland JM, Altman DG. Statistical methods for assessing agreement between two methods of clinical measurement. Lancet 1986;1:307-10.

14 Shrout PE, Fleiss JL. Intraclass correlations: uses in assessing rater reliability. Psychol Bull 1979:86:420-8.

15 Segawa, et al. Factors affecting scanning laser Doppler flwometery. Invest Ophthalmol Vis Sci 1997;38:s774.

16 Hafez AS, et al. Changes in optic nerve head blood flow after therapeutic interocular reduction in glaucoma patients and ocular hypertensives. Ophthalmology 2003;1 10:201-10.

17 Kagemann L, Harris A, Chung HS, et al. Heidelberg retinal flowmetry: factors affecting blood flow measurement. Br J Ophthalmol 1998;82:131-6.

18 Chung HS, Harris A, Kagemann L, et al. Peripapillary retinal blood flow in normal tension glaucoma. Br J Ophthalmol 1999;83:466-9.

19 Nicolela MT, Hnik P, Schulzer M, et al. Reproducibility of retinal and optic nerve head blood flow measurements with scanning laser Doppler flowmetry. J Glaucoma 1997;6:157-64.

20 Sullivan $P$, Cioffi L, Wang $L$, et al. The influence of ocular pulsatility on scanning laser Doppler flowmetry. Am J Ophthalmol 1999;128:81-7.

21 Michelson G, Welzenbach J, Pal I, et al. Automatic full field analysis of perfusion images gained by scanning laser Doppler flowmetry. Br J Ophthalmol 1998;82:1294-300.

22 Michelson G, Welzenbach J, Harazny J. Functional imaging of the retinal microvasculature by scanning laser Doppler flowmetry. Int Ophthalmol $2001 ; 23: 327-35$. 